\title{
Review
}

\section{Risk prediction models for colorectal cancer: A scoping review}

Yasara Manori Samarakoon ${ }^{1}$, Arunasalam Pathmeswaran², Nalika Sepali Gunawardena ${ }^{3}$

${ }^{1}$ National Cancer Control Programme, Ministry of Health, Sri Lanka, ${ }^{2}$ Faculty of Medicine, University of Kelaniya, Sri Lanka,

${ }^{3}$ World Health Organization, Country office for Sri Lanka

\section{Keywords: colorectal cancer, risk prediction model, scoping review, risk predictors, world}

\section{Abstract}

\section{Background and objectives}

Many risk prediction models have been developed globally to identify specific populations at high risk for colorectal cancer in specific settings. Documentation of available evidence from existing studies will serve as a useful information base. We performed a scoping review, to review and analyse published risk prediction models for colorectal cancer the world over.

Methods

A scoping review was undertaken to address the following question 'what are the existing risk prediction models to identify the risk of developing colorectal cancer among individuals in different countries and settings?' using the framework developed by Arksey and O'Malley for scoping reviews. Forty-one articles were included in this review from database searches and from additional searches. The titles and abstracts were reviewed using predetermined screening criteria. We limited our search to existing literature in English language and included both observational and interventional studies.

Results

Out of the 58 risk prediction models identified, most were developed for colorectal cancer followed by advanced colorectal cancer. Most of the articles reviewed were cross sectional studies or cohort studies. Statistical methods such as multiple logistic regression was used by a majority, while few have incorporated non-statistical methods such as consensus method and extracting data from published literature. The authors of the 58 risk prediction models have considered 77 different risk factors excluding the genetic variants.

Conclusions

This comprehensive scoping review demonstrates the capacity of the existing risk models to stratify the general population into risk categories, detailing the studies conducted, location, study design, outcome, overview of the methods, data source and the identified risk predictors. While striving to build on existing knowledge, the review also identifies the research gaps and the need for further improvement.

Corresponding Author: Yasara Manori Samarakoon"<yasara.samarakoon@gmail.com> iD https://orcid.org/0000-0001-6146-9767 Received: October 2017, Accepted revised version December 2017, Published December 2017 Competing Interests: Authors have declared that no competing interests exist

This is an open-access article distributed under a Creative Commons Attribution-Share Alike 4.0 International License (CC BY-SA 4.0), which permits unrestricted use, distribution, and reproduction in any medium, provided the original author and source are attributed and materials are shared under the same license.

\section{Introduction}

Globally, colorectal cancer is ranked as the third most common cancer in men and as the second most common cancer in women. [1]. The tests available to screen for colorectal cancers vary from simple tests, such as the faecal occult blood test to more technical and invasive methods, such as flexible sigmoidoscopy and colonoscopy which have better sensitivity and specificity than other methods [2]. The lifetime risk of having colorectal cancer in a Western country is about $5 \%$ in the population [1]. Thus, screening for colorectal cancer would benefit only this $5 \%$ whilst the remaining $95 \%$ might have to 
undergo this invasive high cost procedure with no personal gain [2]. Evidence from developed countries suggests that it is more efficient to offer colorectal cancer screening using colonoscopy or flexible sigmoidoscopy to high-risk population groups rather than to all as a routine screening test [3]. This has prompted many countries to explore the use of high-risk screening for colorectal cancer with appropriate risk stratification of individuals [4].

With the growing recognition of the potential harms of population-based cancer screening programs, screening based on risk stratification has been proposed as a method of reducing harm as well as a method of focusing on the risk population [5]. If risk-stratified cancer prevention is to be implemented, it requires risk assessment tools that can be used in primary care to identify those most likely to benefit from this intervention [6]. Of the tools to assess the individualized cancer risk, risk prediction models which are simple and can be applied in a community setting by a trained person are considered as useful [7].

Risk prediction modelling is a mechanism which estimates the probability of an individual having a certain condition based on presence of multiple risk factors [7]. An essential feature of a risk prediction model is that it uses multiple predictors to assess individuals regarding their risk of future occurrence of a specific outcome [8]. In the development of risk prediction models, obtaining accurate risk estimates for genetic, environmental and behavioural factors and clinical biological markers etc. becomes important. This is usually achieved via cohort or case-control studies [9]. Furthermore, incorporation of variables from published data and expert opinion is another method of selecting risk predictors $[10,11]$.

There are many risk prediction models developed in different parts of the world to identify specific populations at high risk for colorectal cancer in specific settings. Knowledge regarding the different study designs and statistical methods used is useful for researchers and service providers in the field of colorectal cancer to identify the comprehensiveness and applicability of the various models developed in different parts of the world. Thus, documentation of available evidence from existing studies may serve as a useful information base. In this background, a scoping review was performed, to review and analyse the published risk prediction models for colorectal cancer, the world over. The methodologies used were also reviewed and summarized to facilitate researchers in the field.

\section{Methods}

Scoping reviews are distinguished from systematic reviews in their focus on providing an overview of the research landscape to propose a platform for future research. It differs from a systematic review as it does not evaluate research quality or provide a synthesis or meta-analysis of findings [12]. The present scoping review is conducted with the objective of identifying existing risk prediction models for colorectal cancer. In the methodology, Arksey and O'Malley's (2005) scoping review framework was used. This 
model comprises four key stages which includes, identifying the research question, identifying relevant studies, selecting studies, charting of data and collating, summarizing and reporting results [13].

\section{Identifying the research question}

The review focused on the research question, 'what are the existing risk prediction models to identify the risk of developing colorectal cancer among individuals in different countries and settings?'

\section{Identifying relevant studies}

The review included a search of the scientific literature via PubMed/Medline and Cochrane database. Aligned with the research question, broad search terms were used. These included 'colorectal neoplasm,' 'risk/risk factor/risk assessment,' and 'prediction/model/score.' The search filtered the articles published in peer reviewed journals between $1^{\text {st }}$ of January 2000 to $20^{\text {th }}$ May 2017. Searches were limited to papers in the English language. As guided by Arksey O' Malley's (2005) framework, all articles were screened for relevance to the research question. To capture any missed articles, including those in non-medical databases, a secondary Google Scholar search was initiated that fitted the research question. The selection process is shown in Figure 1.

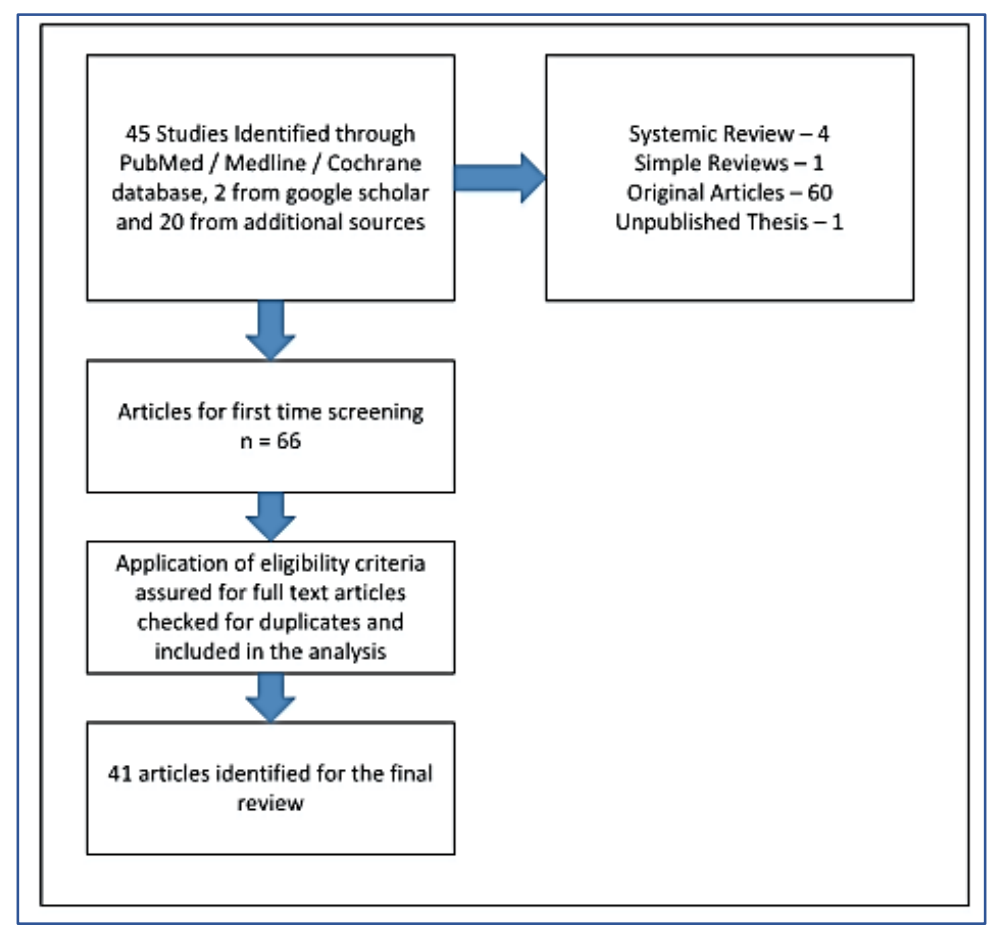

Figure 1: Selection process in the Scoping Review

\section{Selecting studies}

The titles and abstracts were reviewed using predetermined screening criteria. Inclusion and exclusion criteria are listed in Table 1. 
Journal of the Postgraduate Institute of Medicine 2017; 4 (2): E56 1- 24

http://doi.org/10.4038/jpgim.8168

Table 1: Eligibility criteria for selecting studies

\begin{tabular}{|l|l|l|}
\hline Inclusion criteria & 1 & Adult populations more than 18 years of age \\
\hline & 2 & Intervention studies \\
\hline & 3 & Observational studies \\
\hline & 4 & Risk prediction models on behavioral factors \\
\hline & 5 & Risk prediction models on genetic factors \\
\hline & 6 & Models in any setting \\
\hline Exclusion criteria & 7 & $\begin{array}{l}\text { Risk prediction models for either colon, rectal or colorectal } \\
\text { cancer }\end{array}$ \\
\hline & 1 & $\begin{array}{l}\text { Risk prediction models developed for already diagnosed } \\
\text { patients }\end{array}$ \\
\hline
\end{tabular}

Following the identification of the relevant articles, the first author reviewed the full text of each article, confirming the relevance and reviewing overall themes. After applying the screening criteria, forty-one articles were retained for the scoping review. Once the duplicates were removed, the search identified forty-one articles for the final review. Agreement was obtained on overall patterns and gaps.

\section{Charting of data and collating, summarizing and reporting results}

Each of the forty-one articles was charted according to the author (year), study location, study design, outcome of the model, overview of the methods and data source. Because the aim of this scoping review is to identify the existing risk prediction models and does not seek to evaluate quality, charting emphasized the basic characteristics of articles while the validation details of the models (which correspond to the quality of the models) were not reviewed. For studies which included multiple models such as separate models for men and women or for different sub sites, all were included separately.

\section{Results}

\section{Identified risk prediction models}

Forty-one eligible articles were included in the present scoping review and they described fifty-eight risk prediction models. Table 2 summarizes the basic characteristics of the identified risk prediction models. 
Journal of the Postgraduate Institute of Medicine 2017; 4 (2): E56 1- 24

http://doi.org/10.4038/jpgim.8168

Table 2: The basic characteristics of the included articles on colorectal risk prediction models

\begin{tabular}{|c|c|c|c|c|c|c|}
\hline $\begin{array}{l}\text { Authors } \\
\text { (year) }\end{array}$ & $\begin{array}{l}\text { Study } \\
\text { location }\end{array}$ & Study design & Outcome & Overview of methods & Factors Identified & Data Source \\
\hline $\begin{array}{l}\text { 1. Park et al, } 2017 \\
{[14]}\end{array}$ & South Korea & $\begin{array}{l}\text { Hospital based } \\
\text { cross sectional } \\
\text { study }\end{array}$ & $\begin{array}{l}\text { Advanced } \\
\text { colorectal } \\
\text { cancer } \\
\text { (ACRC) }\end{array}$ & $\begin{array}{l}\text { Risk factors were identified from cross } \\
\text { sectional study via multiple logistic } \\
\text { regression. Individual risk factors were } \\
\text { transformed into risk points and overall risk } \\
\text { was calculated by summing up the risk points }\end{array}$ & $\begin{array}{l}\text { Older age, male sex, positive serology } \\
\text { of Helicobacter pylori, high triglyceride } \\
\text { levels, low high-density lipoprotein } \\
\text { levels }\end{array}$ & Medical records \\
\hline $\begin{array}{l}\text { 2. Samarakoon, } \\
2016 \text { [15] }\end{array}$ & Sri Lanka & $\begin{array}{l}\text { Case control } \\
\text { study }\end{array}$ & $\begin{array}{l}\text { Colorectal } \\
\text { cancer } \\
(\mathrm{CRC})\end{array}$ & $\begin{array}{l}\text { Risk factors were identified from a case- } \\
\text { control study via multiple logistic regression } \\
\text { and from consensus method. Individual risk } \\
\text { factors were transformed into risk points and } \\
\text { overall risk was calculated by summing up } \\
\text { the risk points }\end{array}$ & $\begin{array}{l}\text { Older age, frequent deep-fried food, } \\
\text { frequent red meat consumption, } \\
\text { having a first degree relative with } \\
\text { colorectal cancer and/or other cancers } \\
\text { diagnosed at or before } 60 \text { years of } \\
\text { age, history of hypertension for } 10 \\
\text { years, history of inflammatory bowel } \\
\text { disease before } 10 \text { years and history of } \\
\text { polyps before } 10 \text { years }\end{array}$ & $\begin{array}{l}\text { Questionnaire } \\
\text { and medical } \\
\text { records }\end{array}$ \\
\hline $\begin{array}{l}\text { 1. Li et al, } 2015 \\
{[16]}\end{array}$ & China & $\begin{array}{l}\text { Population } \\
\text { based case } \\
\text { control study }\end{array}$ & CRC & $\begin{array}{l}\text { Genetic variants and other non-genetic risk } \\
\text { factors were identified as risk factors via a } \\
\text { case control study. } \\
\text { Multiple models combining genetic and non- } \\
\text { genetic factors were established and receiver } \\
\text { operating characteristic curve analysis was } \\
\text { used to compare the discriminatory power of } \\
\text { different predictive models }\end{array}$ & $\begin{array}{l}\text { Seven } \\
\text { polymorphisms (SNP) as genetic } \\
\text { variants, age, sex, cigarette smoking } \\
\text { and alcohol drinking }\end{array}$ & $\begin{array}{l}\text { Blood tests for } \\
\text { genetics and } \\
\text { Questionnaire }\end{array}$ \\
\hline $\begin{array}{l}\text { 2. Jung et al, } \\
2015 \text { [17] }\end{array}$ & Korea & $\begin{array}{l}\text { Case cohort } \\
\text { design drawn } \\
\text { from an } \\
\text { underlying } \\
\text { large } \\
\text { prospective } \\
\text { cohort study }\end{array}$ & $\begin{array}{l}\text { CRC and } \\
\text { rectal } \\
\text { cancer } \\
(\mathrm{RC})\end{array}$ & $\begin{array}{l}\text { Genetic risk factors were identified in case- } \\
\text { cohort design. A genetic risk score was } \\
\text { calculated by summing the number of risk } \\
\text { alleles over all SNPs based on the cox } \\
\text { proportional hazard regression models. }\end{array}$ & $\begin{array}{l}\text { Seven SNPs identified for colorectal } \\
\text { cancer and rectal cancer excluding } \\
\text { colon cancer alone }\end{array}$ & $\begin{array}{l}\text { Blood tests for } \\
\text { genetics and } \\
\text { Questionnaire }\end{array}$ \\
\hline
\end{tabular}


Journal of the Postgraduate Institute of Medicine 2017; 4 (2): E56 1- 24

http://doi.org/10.4038/jpgim.8168

\begin{tabular}{|c|c|c|c|c|c|c|c|}
\hline & $\begin{array}{l}\text { Schroy et al, } \\
2015 \text { [18] }\end{array}$ & $\begin{array}{l}\text { United } \\
\text { States }\end{array}$ & $\begin{array}{l}\text { Hospital based } \\
\text { cross sectional } \\
\text { study }\end{array}$ & ACRC & $\begin{array}{l}\text { Risk factors were identified from cross } \\
\text { sectional study via multiple logistic } \\
\text { regression. Individual risk factors were } \\
\text { transformed into risk points and overall risk } \\
\text { was calculated by summing up the risk points }\end{array}$ & $\begin{array}{l}\text { Age, smoking, alcohol intake, height, } \\
\text { combined } \quad \text { sex/race/ethnicity } \\
\text { variable }\end{array}$ & Questionnaire \\
\hline & $\begin{array}{l}\text { Steffen et al, } \\
2014 \text { [19] }\end{array}$ & Australia & Cohort study & CRC & $\begin{array}{l}\text { Risk factors identified from cohort study. Risk } \\
\text { prediction equations were developed from } \\
\text { cox proportional hazards regression }\end{array}$ & $\begin{array}{l}\text { Age, sex, body mass index (BMI), } \\
\text { prevalent diabetes, ever having } \\
\text { undergone colorectal cancer } \\
\text { screening, smoking, alcohol intake }\end{array}$ & Questionnaire \\
\hline & $\begin{array}{l}\text { Shin et al, } \\
2014 \text { [20] }\end{array}$ & Korea & $\begin{array}{l}\text { Population } \\
\text { based cross } \\
\text { sectional study }\end{array}$ & CRC & $\begin{array}{l}\text { Risk factors identified from population based } \\
\text { cohort study. Prediction equations } \\
\text { developed from cox-proportional hazard } \\
\text { regression models }\end{array}$ & $\begin{array}{l}\text { Age, BMI, serum cholesterol, family } \\
\text { history of cancer and alcohol } \\
\text { consumption, meat consumption, } \\
\text { height, fasting serum glucose }\end{array}$ & $\begin{array}{l}\text { Questionnaire and } \\
\text { blood test }\end{array}$ \\
\hline & $\begin{array}{l}\text { Shin et al, } \\
2014 \text { [20] }\end{array}$ & Korea & $\begin{array}{l}\text { Population } \\
\text { based cross } \\
\text { sectional study }\end{array}$ & CRC & $\begin{array}{l}\text { Risk factors identified from population based } \\
\text { cohort study. Prediction equations } \\
\text { developed from cox-proportional hazard } \\
\text { regression models }\end{array}$ & $\begin{array}{l}\text { Age, height family history of cancer, } \\
\text { fasting serum glucose and meat } \\
\text { consumption }\end{array}$ & $\begin{array}{l}\text { Questionnaire and } \\
\text { blood test }\end{array}$ \\
\hline & $\begin{array}{l}\text { Shin et al, } \\
2014 \text { [20] }\end{array}$ & Korea & $\begin{array}{l}\text { Population } \\
\text { based cross } \\
\text { sectional study }\end{array}$ & $\mathrm{RC}$ & $\begin{array}{l}\text { Risk factors identified from population based } \\
\text { cohort study. Prediction equations } \\
\text { developed from cox-proportional hazard } \\
\text { regression models }\end{array}$ & $\begin{array}{l}\text { Age, BMI, family history of cancer, } \\
\text { height, fasting serum glucose, total } \\
\text { serum cholesterol, alcohol, meat } \\
\text { consumption }\end{array}$ & $\begin{array}{l}\text { Questionnaire and } \\
\text { blood test }\end{array}$ \\
\hline & $\begin{array}{l}\text { Shin et al, } \\
2014 \text { [20] }\end{array}$ & Korea & $\begin{array}{l}\text { Population } \\
\text { based cross } \\
\text { sectional study }\end{array}$ & $\mathrm{RC}$ & $\begin{array}{l}\text { Risk factors identified from population } \\
\text { based cohort study. Prediction equations } \\
\text { developed from cox-proportional hazard } \\
\text { regression models }\end{array}$ & $\begin{array}{l}\text { Age, family history of cancer, height, } \\
\text { fasting serum glucose, meat } \\
\text { consumption }\end{array}$ & $\begin{array}{l}\text { Questionnaire } \\
\text { and blood test }\end{array}$ \\
\hline & $\begin{array}{l}\text { Shin et al, } \\
2014 \text { [20] }\end{array}$ & Korea & $\begin{array}{l}\text { Population } \\
\text { based cross } \\
\text { sectional study }\end{array}$ & $\begin{array}{l}\text { Colon } \\
\text { cancer } \\
\text { (CC) }\end{array}$ & $\begin{array}{l}\text { Risk factors identified from population based } \\
\text { cohort study. Prediction equations } \\
\text { developed from cox-proportional hazard } \\
\text { regression models }\end{array}$ & $\begin{array}{l}\text { Age, BMI, family history of cancer, } \\
\text { height, fasting serum glucose, total } \\
\text { serum cholesterol, alcohol, meat } \\
\text { consumption }\end{array}$ & $\begin{array}{l}\text { Questionnaire } \\
\text { and blood test }\end{array}$ \\
\hline & $\begin{array}{l}\text { Shin et al, } \\
2014 \text { [20] }\end{array}$ & Korea & $\begin{array}{l}\text { Population } \\
\text { based cross } \\
\text { sectional study }\end{array}$ & CC & $\begin{array}{l}\text { Risk factors identified from population based } \\
\text { cohort study. Prediction equations } \\
\text { developed from cox-proportional hazard } \\
\text { regression models }\end{array}$ & $\begin{array}{l}\text { Age, family history of cancer, height, } \\
\text { fasting serum glucose, meat } \\
\text { consumption }\end{array}$ & $\begin{array}{l}\text { Questionnaire } \\
\text { and blood test }\end{array}$ \\
\hline
\end{tabular}


Journal of the Postgraduate Institute of Medicine 2017; 4 (2): E56 1- 24

http://doi.org/10.4038/jpgim.8168

\begin{tabular}{|c|c|c|c|c|c|c|}
\hline $\begin{array}{l}\text { 11. Tao et al, } 2014 \\
\text { [21] }\end{array}$ & Germany & $\begin{array}{l}\text { Hospital based } \\
\text { cross sectional } \\
\text { study }\end{array}$ & ACRC & $\begin{array}{l}\text { Multiple logistic regression was used to } \\
\text { develop the algorithm. Regression } \\
\text { coefficients based scores were used to } \\
\text { calculate the individual risk }\end{array}$ & $\begin{array}{l}\text { Sex, age, first degree relatives with } \\
\text { history of colorectal cancer, cigarette } \\
\text { smoking, alcohol, red meat } \\
\text { consumption, ever regular use of non- } \\
\text { steroidal anti-inflammatory drugs } \\
\text { (NSAIDS), previous colonoscopy, } \\
\text { previous detection of polyps }\end{array}$ & Questionnaire \\
\hline $\begin{array}{l}\text { 12. Tao et al, } 2014 \\
{[21]}\end{array}$ & Germany & $\begin{array}{l}\text { Hospital based } \\
\text { cross sectional } \\
\text { study }\end{array}$ & CRC & $\begin{array}{l}\text { Multiple logistic regression was used to } \\
\text { develop the algorithm. Regression } \\
\text { coefficients based scores were used to } \\
\text { calculate the individual risk }\end{array}$ & $\begin{array}{l}\text { Sex, age, first degree relatives with } \\
\text { history of colorectal cancer, cigarette } \\
\text { smoking, alcohol, red meat } \\
\text { consumption, ever regular use of } \\
\text { NSAIDS, previous colonoscopy, } \\
\text { previous detection of polyps }\end{array}$ & Questionnaire \\
\hline $\begin{array}{l}\text { 13. Wells et al, } \\
2014 \text { [22] }\end{array}$ & $\begin{array}{l}\text { California } \\
\text { and Hawaii }\end{array}$ & Cohort study & CRC & $\begin{array}{l}\text { Forward stepwise regression was used to } \\
\text { select the most important } \\
\text { variables for use in Cox proportional } \\
\text { regression model for men }\end{array}$ & $\begin{array}{l}\text { Age, BMI, smoking status, first degree } \\
\text { relative with colon cancer, alcohol } \\
\text { consumption, race/ethnicity, years of } \\
\text { education, regular use of aspirin, } \\
\text { multivitamins, red meat intake, } \\
\text { physical activity, history of diabetes }\end{array}$ & Questionnaire \\
\hline $\begin{array}{l}\text { 14. Wells et al, } \\
2014 \text { [22] }\end{array}$ & $\begin{array}{l}\text { California } \\
\text { and Hawaii }\end{array}$ & Cohort study & CRC & $\begin{array}{l}\text { Forward stepwise regression was used to } \\
\text { select the most important } \\
\text { variables for use in Cox proportional } \\
\text { regression model for women }\end{array}$ & $\begin{array}{l}\text { Age, BMI, smoking status, first degree } \\
\text { relative with colon cancer, alcohol } \\
\text { consumption, race/ethnicity, years of } \\
\text { education, regular use of NSAIDS, } \\
\text { multivitamins, use of oestrogen, } \\
\text { history of diabetes }\end{array}$ & Questionnaire \\
\hline $\begin{array}{l}\text { 15. Chen et al, } \\
2014 \text { [23] }\end{array}$ & China & $\begin{array}{l}\text { Hospital based } \\
\text { cross sectional } \\
\text { study }\end{array}$ & ACRC & $\begin{array}{l}\text { Risk factors identified via multiple logistic } \\
\text { regression. Risk scores assigned according to } \\
\text { the beta values of the risk factors }\end{array}$ & $\begin{array}{l}\text { Age, gender, history of coronary heart } \\
\text { disease, egg intake, stool frequency }\end{array}$ & Questionnaire \\
\hline $\begin{array}{l}\text { 16. Kaminski et al, } \\
2014 \text { [24] }\end{array}$ & Poland & $\begin{array}{l}\text { Hospital based } \\
\text { cross sectional } \\
\text { study }\end{array}$ & ACRC & $\begin{array}{l}\text { Risk factors were identified from multiple } \\
\text { logistic regression. Risk level derived from a } \\
\text { scoring method based on regression } \\
\text { coefficients }\end{array}$ & $\begin{array}{l}\text { Age, sex, family history of colorectal } \\
\text { cancer, cigarette smoking, BMI }\end{array}$ & Questionnaire \\
\hline
\end{tabular}


Journal of the Postgraduate Institute of Medicine 2017; 4 (2): E56 1- 24

http://doi.org/10.4038/jpgim.8168

\begin{tabular}{|c|c|c|c|c|c|c|}
\hline $\begin{array}{l}\text { 17. Stegeman et } \\
\text { al, } 2014 \text { [25] }\end{array}$ & Netherlands & $\begin{array}{l}\text { Cross sectional } \\
\text { study }\end{array}$ & ACRC & $\begin{array}{l}\text { Risk questionnaire was developed from a } \\
\text { non-statistical method (undefined). Based on } \\
\text { the risk questionnaire and Faecal } \\
\text { immunochemical testing a risk model was } \\
\text { developed }\end{array}$ & $\begin{array}{l}\text { Age, first degree relative with } \\
\text { colorectal cancer, smoking, faecal } \\
\text { immunochemical test, calcium intake }\end{array}$ & Questionnaire \\
\hline $\begin{array}{l}\text { 18. Stegeman et } \\
\text { al, } 2013 \text { [26] }\end{array}$ & Netherlands & $\begin{array}{l}\text { Cross sectional } \\
\text { study }\end{array}$ & ACRC & $\begin{array}{l}\text { Risk factors were identified from a cross } \\
\text { sectional analysis via bivariate analysis }\end{array}$ & $\begin{array}{l}\text { Age, BMI, gender, first degree relative } \\
\text { with colorectal cancer, menopausal } \\
\text { status (women), smoking, sleep, } \\
\text { vigorous exercise, alcohol, fiber } \\
\text { intake, aspirin/NSAIDS use }\end{array}$ & Questionnaire \\
\hline $\begin{array}{l}\text { 19. Dunlop et al, } \\
2013 \text { [27] }\end{array}$ & Worldwide & $\begin{array}{l}\text { Case-control } \\
\text { studies }\end{array}$ & CRC & $\begin{array}{l}\text { Binary logistic regression was used to identify } \\
\text { the risk predictors }\end{array}$ & $\begin{array}{l}\text { Age, first degree relative } \text { with } \\
\text { colorectal cancer, sex, } 10 \text { SNPs }\end{array}$ & $\begin{array}{l}\text { Questionnaire } \\
\text { and blood test } \\
\text { for genetics }\end{array}$ \\
\hline $\begin{array}{l}\text { 20. Wrong et al, } \\
2013 \text { [28] }\end{array}$ & Hong Kong & $\begin{array}{l}\text { Hospital based } \\
\text { cross sectional } \\
\text { study }\end{array}$ & CRC & $\begin{array}{l}\text { Risk factors were determined from binary } \\
\text { logistic regression }\end{array}$ & $\begin{array}{l}\text { Age, gender, smoking, family history, } \\
\text { BMI, self-reported diabetes, }\end{array}$ & Medical records \\
\hline $\begin{array}{l}\text { 21. Hassan et al, } \\
2013 \text { [29] }\end{array}$ & Italy & $\begin{array}{l}\text { Hospital based } \\
\text { cross sectional } \\
\text { study }\end{array}$ & ACRC & $\begin{array}{l}\text { Risk predictors were determined from } \\
\text { multiple logistic regression }\end{array}$ & Age, gender & Medical records \\
\hline $\begin{array}{l}\text { 22. Johnson et al, } \\
2013 \text { [30] }\end{array}$ & Worldwide & $\begin{array}{l}\text { Risk factors } \\
\text { identified from } \\
\text { published } \\
\text { literature from } \\
\text { meta-analysis }\end{array}$ & CRC & $\begin{array}{l}\text { Random effect } \\
\text { models of the logarithms of risks across the } \\
\text { studies was used to quantify each factor's } \\
\text { impact on colorectal cancer risk }\end{array}$ & $\begin{array}{l}\text { BMI, first degree relative with } \\
\text { colorectal cancer, smoking status, } \\
\text { physical activity, alcohol, } \\
\text { Inflammatory bowel disease, current } \\
\text { or former hormone therapy, } \\
\text { aspirin/NSAIDS, processed meat/red } \\
\text { meat/fruit/vegetable intake }\end{array}$ & Questionnaire \\
\hline $\begin{array}{l}\text { 23. Yarnall et al, } \\
2013 \text { [31] }\end{array}$ & $\begin{array}{l}\text { United } \\
\text { Kindom }\end{array}$ & $\begin{array}{l}\text { Genetic } \\
\text { variants and } \\
\text { environmental } \\
\text { factors were } \\
\text { identified from } \\
\text { literature }\end{array}$ & CRC & $\begin{array}{l}\text { Through a simulation study, using a risk } \\
\text { modelling software risk prediction model } \\
\text { was developed }\end{array}$ & $\begin{array}{l}14 \text { SNPs, BMI, smoking status, alcohol } \\
\text { consumption, red meat consumption, } \\
\text { fiber intake, physical activity }\end{array}$ & $\begin{array}{l}\text { Questionnaire } \\
\text { and blood test } \\
\text { for genetics }\end{array}$ \\
\hline
\end{tabular}


Journal of the Postgraduate Institute of Medicine 2017; 4 (2): E56 1- 24

http://doi.org/10.4038/jpgim.8168

\begin{tabular}{|c|c|c|c|c|c|c|}
\hline $\begin{array}{l}\text { 24. Wang et al, } \\
2013 \text { [32] }\end{array}$ & Taiwan & $\begin{array}{l}\text { Case Control } \\
\text { study and } \\
\text { published } \\
\text { literature }\end{array}$ & CRC & $\begin{array}{l}\text { Prediction model } \\
\text { was constructed by applying Jackknife } \\
\text { feature selection and ANOVA testing }\end{array}$ & 16 SNPS & $\begin{array}{l}\text { Blood test for } \\
\text { genetics }\end{array}$ \\
\hline $\begin{array}{l}\text { 25. Lin et al, } 2013 \\
\text { [33] }\end{array}$ & $\begin{array}{l}\text { United } \\
\text { States }\end{array}$ & Cohort study & ACRC & $\begin{array}{l}\text { Risk predictors and the risk score developed } \\
\text { via a penalized logistic regression method }\end{array}$ & $\begin{array}{l}\text { Age, BMI, smoking, previous } \\
\text { sigmoidoscopy or colonoscopy, } \\
\text { number of first degree relatives with } \\
\text { colorectal cancer, polyp history in past } \\
10 \text { years, physical activity, vegetable } \\
\text { consumption, NSAID use, oestrogen } \\
\text { use }\end{array}$ & Questionnaire \\
\hline $\begin{array}{l}\text { 26. Lubbe et al, } \\
2012 \text { [34] }\end{array}$ & $\begin{array}{l}\text { United } \\
\text { Kingdom }\end{array}$ & $\begin{array}{l}\text { Population } \\
\text { based cross } \\
\text { sectional study }\end{array}$ & CRC & $\begin{array}{l}\text { Risk factors identified from a cross sectional } \\
\text { study. The risk associated with genetic } \\
\text { variants was calculated by unconditional } \\
\text { logistic regression }\end{array}$ & 14 SNPs & $\begin{array}{l}\text { Blood test for } \\
\text { genetics }\end{array}$ \\
\hline $\begin{array}{l}\text { 27. Jo et al, } 2012 \\
\text { [35] }\end{array}$ & Korea & Cohort study & CRC & $\begin{array}{l}\text { Risk predictors were identified from logistic } \\
\text { regression methods. }\end{array}$ & $\begin{array}{l}\text { Age, family history of colorectal } \\
\text { cancer, } 3 \text { SNPs }\end{array}$ & $\begin{array}{l}\text { Questionnaire } \\
\text { and blood test } \\
\text { for genetics }\end{array}$ \\
\hline $\begin{array}{l}\text { 28. Jo et al, } 2012 \\
\text { [35] }\end{array}$ & Korea & Cohort study & CRC & $\begin{array}{l}\text { Risk predictors were identified from logistic } \\
\text { regression methods }\end{array}$ & $\begin{array}{l}\text { Age, family history of colorectal } \\
\text { cancer, } 5 \text { SNPs }\end{array}$ & $\begin{array}{l}\text { Questionnaire } \\
\text { and blood test } \\
\text { for genetics }\end{array}$ \\
\hline $\begin{array}{l}\text { 29. Cai et al, } 2012 \\
{[36]}\end{array}$ & China & $\begin{array}{l}\text { Hospital based } \\
\text { cross sectional } \\
\text { study }\end{array}$ & ACRC & $\begin{array}{l}\text { Risk factors were identified from multiple } \\
\text { logistic regression. Risk level derived from a } \\
\text { scoring method based on regression } \\
\text { coefficients }\end{array}$ & $\begin{array}{l}\text { Age, sex, smoking, diabetes mellitus, } \\
\text { green vegetables, pickled food, fried } \\
\text { food, white meat }\end{array}$ & Questionnaire \\
\hline $\begin{array}{l}\text { 30. Yeoh et al, } \\
2011 \text { [37] }\end{array}$ & Asia & $\begin{array}{l}\text { Hospital based } \\
\text { cross sectional } \\
\text { study }\end{array}$ & ACRC & $\begin{array}{l}\text { Predictors identified from multiple logistic } \\
\text { regression. Risk scores were allocated based } \\
\text { on the odds ratios }\end{array}$ & Age, gender, family history, smoking & Questionnaire \\
\hline $\begin{array}{l}\text { 31. Taylor et al, } \\
2011 \text { [38] }\end{array}$ & $\begin{array}{l}\text { United } \\
\text { States }\end{array}$ & $\begin{array}{l}\text { Population } \\
\text { based cohort } \\
\text { study }\end{array}$ & CRC & $\begin{array}{l}\text { Predictors identified based on the Cox } \\
\text { regression and Harrell's C }\end{array}$ & $\begin{array}{l}\text { Age, first/second/third degree relative } \\
\text { with colorectal cance }\end{array}$ & Questionnaire \\
\hline
\end{tabular}


Journal of the Postgraduate Institute of Medicine 2017; 4 (2): E56 1- 24

http://doi.org/10.4038/jpgim.8168

\begin{tabular}{|c|c|c|c|c|c|c|}
\hline $\begin{array}{l}\text { 32. Marshall et al, } \\
2010 \text { [39] }\end{array}$ & $\begin{array}{l}\text { Canada and } \\
\text { United } \\
\text { States }\end{array}$ & $\begin{array}{l}\text { Cross sectional } \\
\text { study }\end{array}$ & CRC & $\begin{array}{l}\text { Panel performance characteristics and } \\
\text { disease prevalence were used to develop a } \\
\text { scale assessing an individual's current risk of } \\
\text { having colorectal cancer based on } \\
\text { his/her gene signature via multiple logistic } \\
\text { regression }\end{array}$ & Seven genes & $\begin{array}{l}\text { Blood test for } \\
\text { genetics }\end{array}$ \\
\hline $\begin{array}{l}\text { 33. Ma et al, } 2010 \\
\text { [40] }\end{array}$ & Japan & $\begin{array}{l}\text { Population } \\
\text { based Cohort } \\
\text { study }\end{array}$ & CRC & $\begin{array}{l}\text { Risk factors identified from cohort study by } \\
\text { Cox proportion hazard regression }\end{array}$ & $\begin{array}{l}\text { Age, BMI, alcohol, smoking status, } \\
\text { daily physical activity }\end{array}$ & Questionnaire \\
\hline $\begin{array}{l}\text { 34. Ma et al, } 2010 \\
\text { [40] }\end{array}$ & Japan & $\begin{array}{l}\text { Population } \\
\text { based Cohort } \\
\text { study }\end{array}$ & CC & $\begin{array}{l}\text { Risk factors identified from cohort study by } \\
\text { Cox proportion hazard regression }\end{array}$ & $\begin{array}{l}\text { Age, BMI, alcohol, smoking status, } \\
\text { daily physical activity }\end{array}$ & Questionnaire \\
\hline $\begin{array}{l}\text { 35. Ma et al, } 2010 \\
\text { [40] }\end{array}$ & Japan & $\begin{array}{l}\text { Population } \\
\text { based Cohort } \\
\text { study }\end{array}$ & $\mathrm{RR}$ & $\begin{array}{l}\text { Risk factors identified from cohort study by } \\
\text { Cox proportion hazard regression }\end{array}$ & $\begin{array}{l}\text { Age, BMI, alcohol, smoking status, } \\
\text { daily physical activity }\end{array}$ & Questionnaire \\
\hline $\begin{array}{l}\text { 36. Bener et al, } \\
2010 \text { [41] }\end{array}$ & Qatar & $\begin{array}{l}\text { Hospital based } \\
\text { case-control } \\
\text { study }\end{array}$ & CRC & $\begin{array}{l}\text { Risk predictors developed from multivariate } \\
\text { stepwise logistic regression }\end{array}$ & $\begin{array}{l}\text { Family history of colorectal cancer, } \\
\text { BMI, smoking status, soft drinks, } \\
\text { bakery products }\end{array}$ & Questionnaire \\
\hline $\begin{array}{l}\text { 37. Wei et al, } 2009 \\
\text { [42] }\end{array}$ & $\begin{array}{l}\text { United } \\
\text { States }\end{array}$ & Cohort study & CC & $\begin{array}{l}\text { Risk factors derived from cohort study. } \\
\text { Relative risk based on cumulative incidence } \\
\text { of colon cancer by the age of } 70 \text { years. Colon } \\
\text { cancer risk was determined by multivariate } \\
\text { non-linear poison regression }\end{array}$ & $\begin{array}{l}\text { Smoking, consistent high relative } \\
\text { weight, daily consumption of red or } \\
\text { processed meat, low physical activity } \\
\text { level, being never screened low daily } \\
\text { folate, current or past HRT, first } \\
\text { degree relative with colon cancer, } \\
\text { aspirin use, }\end{array}$ & Questionnaire \\
\hline $\begin{array}{l}\text { 38. Almurshed et } \\
\text { al, } 2009 \text { [43] }\end{array}$ & Saudi Arabia & $\begin{array}{l}\text { Hospital-based } \\
\text { case control } \\
\text { study }\end{array}$ & CRC & $\begin{array}{l}\text { Risk factors were identified from a case } \\
\text { control study and the model was developed } \\
\text { from multiple logistic regression }\end{array}$ & $\begin{array}{l}\text { Region, education level, marital status, } \\
\text { employment status, physical activity, } \\
\text { activity level, knowledge on high-fiber } \\
\text { diet }\end{array}$ & Questionnaire \\
\hline
\end{tabular}


Journal of the Postgraduate Institute of Medicine 2017; 4 (2): E56 1- 24

http://doi.org/10.4038/jpgim.8168

\begin{tabular}{|c|c|c|c|c|c|c|}
\hline $\begin{array}{l}\text { 39. Kastrinos et al, } \\
2009 \text { [44] }\end{array}$ & $\begin{array}{l}\text { United } \\
\text { States }\end{array}$ & $\begin{array}{l}\text { Hospital based } \\
\text { cross sectional } \\
\text { study }\end{array}$ & CRC & $\begin{array}{l}\text { Risk assessed from an algorithm of three } \\
\text { questions developed from recursive } \\
\text { partitioning analysis }\end{array}$ & $\begin{array}{l}\text { First degree relative with colorectal } \\
\text { cancer or Lynch syndrome related } \\
\text { cancer diagnosed before } 50 \text { years of } \\
\text { age, colorectal cancer or polyps } \\
\text { diagnosed before } 50 \text { years of age, } \\
\text { having three or more relatives } \\
\text { diagnosed with colorectal cancer }\end{array}$ & $\begin{array}{l}\text { Simple three } \\
\text { question model }\end{array}$ \\
\hline $\begin{array}{l}\text { 40. Freedman et } \\
\text { al, } 2009 \text { [45] }\end{array}$ & $\begin{array}{l}\text { United } \\
\text { States }\end{array}$ & $\begin{array}{l}\text { Population } \\
\text { based case } \\
\text { control study }\end{array}$ & Distal CC & $\begin{array}{l}\text { Risk factors were identified from the case } \\
\text { control studies. Relative risks and } \\
\text { attributable risks were combined with the } \\
\text { baseline age specific cancer hazard rates and } \\
\text { the mortality rates to estimate the individual } \\
\text { risk }\end{array}$ & $\begin{array}{l}\text { BMI, number of first degree relatives } \\
\text { with colorectal cancer, polyp history } \\
\text { for the last } 10 \text { years, history of } \\
\text { previous } \\
\text { sigmoidoscopy/colonoscopy for the } \\
\text { last } 10 \text { years, NSAIDS use }\end{array}$ & Questionnaire \\
\hline $\begin{array}{l}\text { 41. Freedman et } \\
\text { al, } 2009 \text { [45] }\end{array}$ & $\begin{array}{l}\text { United } \\
\text { States }\end{array}$ & $\begin{array}{l}\text { Population } \\
\text { based case } \\
\text { control study }\end{array}$ & Distal CC & $\begin{array}{l}\text { Risk factors were identified from the case } \\
\text { control studies. Relative risks and } \\
\text { attributable risks were combined with the } \\
\text { baseline age specific cancer hazard rates and } \\
\text { the mortality rates to estimate the individual } \\
\text { risk }\end{array}$ & $\begin{array}{l}\text { Cancer-negative } \\
\text { sigmoidoscopy/colonoscopy in the } \\
\text { last } 10 \text { years, polyp history in the last } \\
10 \text { years, history of colorectal cancer } \\
\text { in first degree relatives, aspirin and } \\
\text { NSAIDS drug use, oestrogen exposure } \\
\text { in last two years }\end{array}$ & Questionnaire \\
\hline $\begin{array}{l}\text { 42. Freedman et } \\
\text { al, } 2009 \text { [45] }\end{array}$ & $\begin{array}{l}\text { United } \\
\text { States }\end{array}$ & $\begin{array}{l}\text { Population } \\
\text { based case } \\
\text { control study }\end{array}$ & $\begin{array}{l}\text { Proximal } \\
\text { CC }\end{array}$ & $\begin{array}{l}\text { Risk factors were identified from the case } \\
\text { control studies. Relative risks and } \\
\text { attributable risks were combined with the } \\
\text { baseline age specific cancer hazard rates and } \\
\text { the mortality rates to estimate the individual } \\
\text { risk }\end{array}$ & $\begin{array}{l}\text { Cancer-negative } \\
\text { sigmoidoscopy/colonoscopy in the } \\
\text { last } 10 \text { years, polyp history in the last } \\
10 \text { years, history of CRC in first degree } \\
\text { relatives, aspirin and NSAIDS use, } \\
\text { cigarette smoking, BMI, vegetable } \\
\text { consumption }\end{array}$ & Questionnaire \\
\hline
\end{tabular}


Journal of the Postgraduate Institute of Medicine 2017; 4 (2): E56 1- 24

http://doi.org/10.4038/jpgim.8168

\begin{tabular}{|c|c|c|c|c|c|c|}
\hline $\begin{array}{l}\text { 43. Freedman et } \\
\text { al, } 2009 \text { [45] }\end{array}$ & $\begin{array}{l}\text { United } \\
\text { States }\end{array}$ & $\begin{array}{l}\text { Population } \\
\text { based case } \\
\text { control study }\end{array}$ & $\begin{array}{l}\text { Proximal } \\
\text { CC }\end{array}$ & $\begin{array}{l}\text { Risk factors were identified from the case } \\
\text { control studies. Relative risks and } \\
\text { attributable risks were combined with the } \\
\text { baseline age specific cancer hazard rates and } \\
\text { the mortality rates to estimate the individual } \\
\text { risk }\end{array}$ & $\begin{array}{l}\text { Cancer-negative } \\
\text { sigmoidoscopy/colonoscopy in the } \\
\text { last } 10 \text { years, polyp history in the last } \\
10 \text { years, history of colorectal cancer } \\
\text { in first degree relatives, aspirin and } \\
\text { NSAID use, current leisure-time } \\
\text { vigorous activity, vegetable } \\
\text { consumption, oestrogen exposure for } \\
\text { last } 2 \text { years }\end{array}$ & Questionnaire \\
\hline $\begin{array}{l}\text { 44. Freedman et } \\
\text { al, } 2009 \text { [45] }\end{array}$ & $\begin{array}{l}\text { United } \\
\text { States }\end{array}$ & $\begin{array}{l}\text { Population } \\
\text { based case } \\
\text { control study }\end{array}$ & $\mathrm{RC}$ & $\begin{array}{l}\text { Risk factors were identified from the case } \\
\text { control studies. Relative risks and } \\
\text { attributable risks were combined with the } \\
\text { baseline age specific cancer hazard rates and } \\
\text { the mortality rates to estimate the individual } \\
\text { risk }\end{array}$ & $\begin{array}{l}\text { Cancer-negative } \\
\text { sigmoidoscopy/colonoscopy in the } \\
\text { last } 10 \text { years, polyp history in the last } \\
10 \text { years, history of CRC in first degree } \\
\text { relatives, aspirin and NSAID use, } \\
\text { current leisure-time vigorous activity }\end{array}$ & Questionnaire \\
\hline $\begin{array}{l}\text { 45. Freedman et } \\
\text { al, } 2009 \text { [45] }\end{array}$ & $\begin{array}{l}\text { United } \\
\text { States }\end{array}$ & $\begin{array}{l}\text { Population } \\
\text { based case } \\
\text { control study }\end{array}$ & RR & $\begin{array}{l}\text { Risk factors were identified from the case } \\
\text { control studies. Relative risks and } \\
\text { attributable risks were combined with the } \\
\text { baseline age specific cancer hazard rates and } \\
\text { the mortality rates to estimate the individual } \\
\text { risk }\end{array}$ & $\begin{array}{l}\text { Cancer-negative } \\
\text { sigmoidoscopy/colonoscopy in the } \\
\text { last } 10 \text { years, polyp history in the last } \\
10 \text { years, history of colorectal cancer } \\
\text { in first degree relatives, aspirin and } \\
\text { NSIAD use, BMI, current leisure-time } \\
\text { vigorous activity, oestrogen exposure } \\
\text { in last two years }\end{array}$ & Questionnaire \\
\hline $\begin{array}{l}\text { 46. Liu et al, } 2008 \\
\text { [46] }\end{array}$ & Taiwan & $\begin{array}{l}\text { Population } \\
\text { based case } \\
\text { control study }\end{array}$ & CRC & $\begin{array}{l}\text { Multivariate logistic regression was used to } \\
\text { identify the risk factors. Individual risk factors } \\
\text { were transformed into risk points and overall } \\
\text { risk was calculated by summing up the risk } \\
\text { points }\end{array}$ & $\begin{array}{l}\text { Race, occupation, physical activity, } \\
\text { coffee intake, consumption of white } \\
\text { meat, seafood, vegetables and fruits, } \\
\text { method of cooking of meat, alcohol } \\
\text { consumption in males only }\end{array}$ & Questionnaire \\
\hline $\begin{array}{l}\text { 47. Han et al, } 2008 \\
{[47}\end{array}$ & Not given & Not defined & CRC & $\begin{array}{l}\text { Logistic regression was used to identify the } \\
\text { genetic predictors for colorectal cancer }\end{array}$ & Five genes & $\begin{array}{l}\text { Blood test for } \\
\text { genetics }\end{array}$ \\
\hline
\end{tabular}


Journal of the Postgraduate Institute of Medicine 2017; 4 (2): E56 1- 24

http://doi.org/10.4038/jpgim.8168

\begin{tabular}{|c|c|c|c|c|c|c|}
\hline $\begin{array}{l}\text { 48. Driver et al, } \\
2007 \text { [48] }\end{array}$ & $\begin{array}{l}\text { United } \\
\text { States }\end{array}$ & $\begin{array}{l}\text { Prospective } \\
\text { cohort study }\end{array}$ & CRC & $\begin{array}{l}\text { Logistic regression was used to determine } \\
\text { the independent predictors of incident } \\
\text { colorectal cancer over the follow-up period. } \\
\text { Risk scores were created from the sum of the } \\
\text { odds ratios and used to divide the cohort into } \\
\text { categories of increasing relative risk }\end{array}$ & Age, history of smoking, BMI & Medical Records \\
\hline $\begin{array}{l}\text { 49. Driver et al, } \\
2007 \text { [48] }\end{array}$ & $\begin{array}{l}\text { United } \\
\text { States }\end{array}$ & $\begin{array}{l}\text { Prospective } \\
\text { cohort study }\end{array}$ & CC & $\begin{array}{l}\text { Logistic regression was used to determine } \\
\text { the independent predictors of incident } \\
\text { colorectal cancer over the follow-up period. } \\
\text { Risk scores were created from the sum of the } \\
\text { odds ratios and used to divide the cohort into } \\
\text { categories of increasing relative risk }\end{array}$ & $\begin{array}{l}\text { Age, weekly or daily alcohol use, } \\
\text { smoking status, BMl }\end{array}$ & Medical Records \\
\hline $\begin{array}{l}\text { 50. Lin et al, } 2006 \\
\text { [49] }\end{array}$ & $\begin{array}{l}\text { United } \\
\text { States }\end{array}$ & $\begin{array}{l}\text { Predetermined } \\
\text { risk factors } \\
\text { were used to } \\
\text { develop the } \\
\text { model }\end{array}$ & CRC & $\begin{array}{l}\text { Predetermined risk factors were given scores } \\
\text { based on a previously validated scoring } \\
\text { system }\end{array}$ & $\begin{array}{l}\text { Age, sex, first degree relative with } \\
\text { colorectal cancer or second degree } \\
\text { relative with adenoma }\end{array}$ & Questionnaire \\
\hline $\begin{array}{l}\text { 51. Wei et al, } 2004 \\
\text { [50] }\end{array}$ & $\begin{array}{l}\text { United } \\
\text { States }\end{array}$ & Cohort studies & CC & $\begin{array}{l}\text { Risk factors were identified from cohort } \\
\text { studies and the risk prediction model was } \\
\text { developed based on the pooled logistic } \\
\text { regression to identify the multivariate risk } \\
\text { predictors }\end{array}$ & $\begin{array}{l}\text { Age, BMI, sex, alcohol, smoking, first } \\
\text { degree relative with colon cancer, } \\
\text { height, physical activity, processed } \\
\text { meat, pork/lamb, servings of beef } \\
\text { calcium intake, folate intake, }\end{array}$ & Questionnaire \\
\hline $\begin{array}{l}\text { 52. Wei et al, } 2004 \\
\text { [50] }\end{array}$ & $\begin{array}{l}\text { United } \\
\text { States }\end{array}$ & Cohort studies & RR & $\begin{array}{l}\text { Risk factors were identified from cohort } \\
\text { studies and the risk prediction model was } \\
\text { developed based on the pooled logistic } \\
\text { regression to identify the multivariate risk } \\
\text { predictors }\end{array}$ & $\begin{array}{l}\text { Age, BMI, sex, alcohol, smoking, first } \\
\text { degree relative with colon cancer, } \\
\text { height, physical activity, processed } \\
\text { meat, pork/lamb, servings of beef } \\
\text { calcium intake, folate intake, }\end{array}$ & Questionnaire \\
\hline $\begin{array}{l}\text { 53. Imperiale et al, } \\
2003 \text { [51] }\end{array}$ & $\begin{array}{l}\text { United } \\
\text { States }\end{array}$ & $\begin{array}{l}\text { Cross sectional } \\
\text { study }\end{array}$ & ACRC & $\begin{array}{l}\text { A clinical index of three variables were } \\
\text { created based on the information of persons. } \\
\text { Risk score was created by allocating points to } \\
\text { variables (method for scoring undefined) }\end{array}$ & $\begin{array}{l}\text { Age, sex, distal findings of } \\
\text { colonoscopy }\end{array}$ & Questionnaire \\
\hline
\end{tabular}


Journal of the Postgraduate Institute of Medicine 2017; 4 (2): E56 1- 24

http://doi.org/10.4038/jpgim.8168

\begin{tabular}{|c|c|c|c|c|c|c|}
\hline $\begin{array}{l}\text { 54. Betes et al, } \\
2003 \text { [52] }\end{array}$ & Spain & $\begin{array}{l}\text { Hospital based } \\
\text { cross sectional } \\
\text { study }\end{array}$ & CRC & $\begin{array}{l}\text { Risk factors and the risk score was developed } \\
\text { from multiple logistic regression based on } \\
\text { the results from the cross-sectional study }\end{array}$ & Age, BMI, gender & Medical records \\
\hline $\begin{array}{l}\text { 55. Camp et al, } \\
2002 \text { [53] }\end{array}$ & $\begin{array}{l}\text { United } \\
\text { States }\end{array}$ & $\begin{array}{l}\text { Population } \\
\text { based case- } \\
\text { control study }\end{array}$ & CC & $\begin{array}{l}\text { Classification tree analysis was used to } \\
\text { identify the interactions between risk factors }\end{array}$ & $\begin{array}{l}\text { Age, first degree relative with } \\
\text { colorectal cancer, BMI, NSAID use, } \\
\text { long term vigorous activity, western } \\
\text { diet, calcium intake, lutein intake, folic } \\
\text { acid, refined grain intake, prudent } \\
\text { dietary pattern }\end{array}$ & Questionnaire \\
\hline $\begin{array}{l}\text { 56. Colditz et al, } \\
2000 \text { [54] }\end{array}$ & $\begin{array}{l}\text { United } \\
\text { States }\end{array}$ & $\begin{array}{l}\text { Consensus } \\
\text { process }\end{array}$ & $\mathrm{CC}$ & $\begin{array}{l}\text { Risk factors identified from group consensus } \\
\text { and relative risks identified from different } \\
\text { studies. Risk points were allocated according } \\
\text { to the strength of the causal association and } \\
\text { summed. Population average risk of cancer } \\
\text { and cumulative } 10 \text {-year risk was obtained } \\
\text { from SEER data. Individual ranking relative to } \\
\text { the population average was determined. The } \\
\text { risk was evaluated for validity using colon } \\
\text { cancer incidence in prospective cohort data. }\end{array}$ & $\begin{array}{l}\text { Family history of colon, BMI, screening } \\
\text { (FOBT and sigmoidoscopy), aspirin, } \\
\text { inflammatory bowel disease, folate, } \\
\text { vegetables, alcohol, height, physical } \\
\text { activity, oestrogen replacement, Oral } \\
\text { contraceptives, red meat, fruits, } \\
\text { fiber, saturated fat, } \\
\text { cigarette smoking }\end{array}$ & Questionnaire \\
\hline
\end{tabular}


Among the identified models, 29 have colorectal cancer, 12 have advanced colorectal cancer (defined as either having an invasive cancer, an adenoma of $10 \mathrm{~mm}$ or more, a villous adenoma or having an adenoma with high grade dysplasia), 11 have colon cancer and six, rectal cancer as the outcome.

\section{Socio-demographic characteristics of the study populations}

A majority of the risk prediction models have been developed in United States of America $[18,22,33,38,39,42,44,45,48,49,50,51,53,54]$ followed by Korea [14, 17, 20, 35]. Three models have been developed in China $[16,23,36]$ and Japan [40] while, Netherlands [25, 26], Germany [21], Taiwan[32, 46] and United Kingdom [31, 34] have developed two models each. Many other countries, such as Australia [19], Poland [24], Hong Kong [28], Italy [29], Qatar [41], Saudi Arabia [43], Spain [52] and Sri Lanka [15] have also developed country-specific risk prediction models for colorectal cancer. When considering the global approach two models have been developed globally $[27,30]$ while another has been developed for Asia [37].

Most of the risk prediction models address both men and women. However, 11 models are specific for females [20, 22, 35, 42, 45, 50] while 10 models are specific for males [20, $22,35,45,48]$. Although many models have not restricted application to a specific age limit, 11 models have been developed from populations aged 30 years and above [15, 20, $42,43,50], 10$ from populations aged 50 years and above $[18,25,26,45,51]$ and seven from populations aged 40 years and above [14, 19, 23, 24, 36, 38, 52]. In addition, one model has used a population above 20 years [35] while another, populations lower than 80 years [34].

\section{Development of the models}

Determination of risk factors was performed via various study designs in these risk prediction models. A total of twenty-three were developed from hospital based $(n=14)$ or population based $(n=9)$ cross sectional studies in participants undergoing screening colonoscopy, while another 13 models were developed from case control studies. The cases were identified from hospitals $(n=6)$ or population registries $(n=7)$ while controls were identified from hospitals, primary care or from the community. Sixteen models were developed using cohort designs where most of the cases were identified through cancer registries over a period of follow-up. Four risk prediction models were developed from reviewing published literature $[30,31,32,49]$, while one model was developed using a consensus procedure [54].

A total of six models include only variables that are routinely available in the medical records $[14,28,29,48,52]$. A majority $(n=35)$ of models include variables obtained through a questionnaire $[18,19,21,22,23,24,25,26,27,28,30,33,36,37,38,39,40,41,42,43$, $44,45,46,49,50,51,53,54]$. The questionnaires range from those with only one or two simple questions to those with detailed questions on factors such as diet, physical activity, alcohol and smoking habits and past medical facts. Three purely genetic models have used only a blood test for genetic biomarkers [32, 34, 39], while thirteen models have 
used data from a questionnaire and the results of blood tests for genetic biomarkers and other biochemical tests $[16,17,20,27,31,35,47]$. One model has obtained data both from a questionnaire and medical records [15].

The prominent method of developing the risk prediction models was via statistical methods. A majority of the studies have used multiple logistic regression to identify the risk predictors $[16,27,28,30,33,34,35,41,43,47,50,52]$ followed by the allocation of the risk points, based on the values of beta-coefficients $[14,15,18,21,23,24,36,37,39$, $45,46,48]$. Fourteen models have incorporated Cox proportional hazards regression to develop the risk model and the score [17, 19, 20, 22, 38, 40]. One model [30] was developed from meta-analysis of various studies, one [31] used risk modelling software in a simulated population and one [26] used pure bivariate analysis. Several other statistical methods were used in the development of models such as jackknife feature selection and ANOVA testing [32], multivariate non-linear Poisson regression [42], recursive portioning analysis [44] and classification tree analysis [53]. However, four models were identified as developed from non-statistical methods such as consensus method $[25,51]$, extracting data from previously published validated models [49] or from previous studies [54].

\section{Risk predictors in the developed models}

The risk prediction models identified can be broadly categorized as non-genetic, genetic and mixed models with both genetic and non-genetic predictors. Among the models four were purely genetic $[17,32,34,47]$, seven models have both components $[16,27,31,35$, 39 ] while the rest of the models are non-genetic $(n=48)$. The authors of the 58 risk prediction models have considered 77 different risk factors (excluding genetic factors) as shown in Table 3.

Table 3: Seventy-seven risk factors identified across all included studies (excluding genetic factors)

\begin{tabular}{|l|l|}
\hline Socio-demographic characteristics & Diet \\
\hline Age & Meat consumption \\
\hline Male Sex & Red meat consumption \\
\hline Gender & Deep fried food consumption \\
\hline Sex/race/ethnicity variable & Egg intake \\
\hline Race/ethnicity & Calcium intake \\
\hline Years of education/education level & Fiber intake \\
\hline Region & Processed meat intake \\
\hline Marital status & Fruits intake \\
\hline Employment status & Vegetable intake \\
\hline Anthropometric measurements & Green vegetable intake \\
\hline Height & Prickled food intake \\
\hline Consistent high relative weight & Fried food intake \\
\hline BMI & White meat intake \\
\hline Genetic characteristics & Soft drinks intake \\
\hline Family history of cancer & Bakery products intake \\
\hline
\end{tabular}




\begin{tabular}{|c|c|}
\hline $\begin{array}{l}\text { First degree relatives with history of colorectal } \\
\text { cancer }\end{array}$ & Low daily folate intake \\
\hline $\begin{array}{l}\text { First degree relatives with history of other } \\
\text { cancer }\end{array}$ & Coffee intake \\
\hline Relatives with colon cancer & Seafood intake \\
\hline $\begin{array}{l}\text { First/second/third degree relative with } \\
\text { colorectal cancer }\end{array}$ & Pork/lamb intake \\
\hline $\begin{array}{l}\text { First degree relatives with Lynch Syndrome } \\
\text { related cancer diagnosed before } 50 \text { years }\end{array}$ & Servings of beef \\
\hline $\begin{array}{l}\text { Having three or more relatives diagnosed with } \\
\text { colorectal cancer }\end{array}$ & Western diet \\
\hline Second degree relatives with adenoma & Lutein intake \\
\hline Personal Medical History & Refined grain intake \\
\hline Diabetes/History of diabetes & Prudent dietary pattern \\
\hline $\begin{array}{l}\text { History of hypertension/History of hypertension } \\
\text { for } 10 \text { years }\end{array}$ & Female hormonal factors \\
\hline History of inflammatory bowel disease & Use of oestrogen \\
\hline History of coronary heart disease & Menopausal status \\
\hline Stool frequency & Current or former hormone therapy \\
\hline Self-reported diabetes & Current or past HRT \\
\hline History of polyps & Drugs \\
\hline Polyp history for last 10 years & $\begin{array}{l}\text { Ever regular use of non-steroidal anti- } \\
\text { inflammatory drugs }\end{array}$ \\
\hline Biomarkers & Regular use of aspirin \\
\hline Positive serology of Helicobacter pylori & Regular use of multivitamins \\
\hline High triglyceride levels & Aspirin/NSAID use \\
\hline Low high-density lipoprotein level & Lifestyle related factors \\
\hline Total serum cholesterol & Cigarette smoking \\
\hline Fasting serum glucose & Alcohol drinking \\
\hline Other tests & Physical activity \\
\hline Prior sigmoidoscopy or colonoscopy & Sleep \\
\hline Faecal immunochemical test & Vigorous exercise \\
\hline Distal findings of colonoscopy & Activity level \\
\hline Faecal occult blood test & Current leisure time vigorous activity \\
\hline Personal Medical History & Method of cooking of meat \\
\hline \multicolumn{2}{|l|}{ Diabetes/History of diabetes } \\
\hline \multicolumn{2}{|l|}{$\begin{array}{l}\text { History of hypertension/History of hypertension } \\
\text { for } 10 \text { years }\end{array}$} \\
\hline \multicolumn{2}{|l|}{ History of inflammatory bowel disease } \\
\hline \multicolumn{2}{|l|}{ History of coronary heart disease } \\
\hline \multicolumn{2}{|l|}{ Stool frequency } \\
\hline \multicolumn{2}{|l|}{ Self-reported diabetes } \\
\hline \multicolumn{2}{|l|}{ History of polyps } \\
\hline Polyp history for last 10 years & \\
\hline
\end{tabular}




\section{Discussion}

A comprehensive review was performed that identified 58 risk prediction models in fortyone studies. This scoping review demonstrates that multiple risk prediction models exist for predicting the risk of developing colorectal cancer, advanced colorectal cancer, colon cancer and rectal cancer among asymptomatic male and female population groups. A majority had been developed using data from analytical cross-sectional studies. The other contributions are from case-control studies and cohort studies, in this order. Though many have used multiple logistic regression statistical methods in developing the model, a minority have incorporated non-statistical methods such as consensus processes and reviewing literature. The identified models ranged from pure non-genetic models to pure genetic models including a small number of models with both components.

The main strength of this review is the extensive search strategy and careful screening of the studies applicable to the research question. Use of a broad search strategy has allowed us to identify many more risk models than reported in previous reviews in the area of risk prediction in colorectal cancer. Therefore, this review is more comprehensive and up to date. However, the inclusion criteria included only asymptomatic individuals, excluding symptomatic and already diagnosed populations, limiting the applicability of models for those with familial syndromes such as hereditary non-polyposis colorectal cancer or familial adenomatous polyposis. Furthermore, since the research question in this review was to identify existing models, the performance of the risk prediction models was not evaluated with respect to their discriminative power and calibration properties which is a drawback as the usefulness of the models in terms of validity could not be shown.

This scoping review demonstrates that the existing risk models have the capacity to stratify the general population into risk categories. Risk stratification applied through these models can help to identify the populations who may benefit from invasive screening preventing those at low risk of disease from being exposed to the direct and indirect harms of screening procedures. This may also address the issues of cost effectiveness of screening programmes using colonoscopy since this risk stratification can limit the number of individuals referred for screening. The use of risk prediction models can also increase the screening behaviour of the public as well as provide an opportunity to encourage lifestyle changes.

However, several challenges can be anticipated when implementing the existing models in clinical practice. Many require collection of dietary information using food frequency questionnaires. Though these can be used to generate accurate estimates in the research setting, practical applicability at population level is questionable. With assessment of lifetime physical activity, recall bias becomes an issue. Furthermore, information collected other than from routine medical reports, becomes questionable with regards to accuracy. On the other hand, access to medical reports also becomes a practical 
challenge when applying these modules at community level. Furthermore, models including genetic variants, require blood sample collection as well as processing which is not so user friendly or feasible at population level, in addition to increased cost.

It is necessary to evaluate the performance of these models with respect to their discriminative and calibration properties. Evaluation of the utility of these models in. The role of the currently available models in clinical practice will be defined when comparative data on the performance of different models becomes available. However, the choice of which risk model is applicable to each country will be based on validation studies in the population of interest. Furthermore, research is needed to identify optimal implementation strategies, where feasibility, accessibility, cost-effectiveness and impact on morbidity and mortality in comparison to the already existing programmes is assessed. Finally, it is necessary to assess the advantages and disadvantages of implementing these risk models in clinical practice via randomized controlled trials.

\section{Conclusions}

This comprehensive and up-to-date scoping review describes this emergent body of literature, detailing the studies conducted, location, study design, outcome of the model, overview of the methods, data source and risk predictors identified, demonstrating the capacity of the existing risk models to stratify the general population into risk categories. While striving to build on existing knowledge, the review also identifies the research gaps and the need for further improvement.

\section{References}

1. World Health Organization. GLOBOCAN 2012, Colorectal Cancer: Estimated Incidence, Mortality and Prevalence Worldwide in 2012. 2015; 1:19. Available from http://globocan.iarc.fr/Pages/fact_sheets_cancer.aspx\#

2. Bretthauer M. Colorectal cancer screening. J Intern Med. 2011; 270: 87-98. doi: 10.1111/j.1365-2796.2011.02399.x https://doi.org/10.1111/j.1365-2796.2011.02399.x

3. Boyle P, Leon ME. Epidemiology of colorectal cancer. Br Med Bull. 2002;64: 1-25. https://doi.org/10.1093/bmb/64.1.1 PMid:12421722

4. 4. Nelson RS, Thorson AG. Colorectal cancer screening. Curr Oncol Rep. 2009;11(6): 482-489 https://doi.org/10.1007/s11912-009-0065-8

5. Burton H, Chowdhury S, Dent T, Hall A, Pashayan N, Pharoah P. Public health implications from COGS and potential for risk stratification and screening. Nat Genet. 2013;45(4):349-351 https://doi.org/10.1038/ng.2582 PMid:23535723

6. Armstrong R, Hall BJ, Doyle J, Waters E. Scoping the scope of a Cochrane review. Journal of J Public Health. 2011; 33: 147-150. https://doi.org/10.1093/pubmed/fdr015 PMid:21345890 
7. Win, A.K., Macinnis, R.J., Hopper, J.L., Jenkins, M.A. (2011). Risk Prediction Models for Colorectal Cancer: A Review. Cancer Epidemiol Biomarkers Prev. 21 (3):398410. https://doi.org/10.1158/1055-9965.EPI-11-0771 PMid:22169185

8. Vickers AJ. Prediction Models in Cancer Care. CA Cancer J Clin. 2011; 6(5): 315. https://doi.org/10.3322/caac.20118

9. Freedman AN, Seminara D, Gail MH, Hartge P, Colditz GA, Ballard-Barbash R, et alCancer risk prediction models: a workshop on development, evaluation and application. J Natl Cancer Inst. 2005; 97(10): 715-723. https://doi.org/10.1093/jnci/dji128 PMid:15900041

10. Kumari, PBVR. Risk factors and risk assessment of Breast Cancer among women in the district of Colombo. MD Thesis, Faculty of Medicine, University of Colombo Sri Lanka. 2013.

11. Tammemagi CM, Pinsky PF, Caporaso NE, Kyale PA, Hocking WG, Church TR, et al. Lung Cancer Risk Prediction: Prostate, Lung, Colorectal and Ovarian Cancer Screening Trial Models and Validation. J Natl Cancer Inst. 2011; 103:1058-1068. https://doi.org/10.1093/jnci/djr173 PMid:21606442 PMCid:PMC3131220

12. Gough D, Thomas J, Oliver S. Clarifying differences between review designs and methods. Syst. Rev. 2012; 1: 28. https://doi.org/10.1186/2046-4053-1-28 PMid:22681772 PMCid:PMC3533815

13. Arksey H, O'Malley L. Scoping studies: towards a methodological framework. Int J Soc Res Methodol. 2005;8(1):19-32. https://doi.org/10.1080/1364557032000119616

14. Park YM, Kim HS, Park JJ, Baik SJ, Youn YH, Kim J, et al. A simple scoring model for advanced colorectal neoplasm in asymptomatic subjects aged $40-49$ years. BMC Gastroenterol. 2017; 17:7. DOI 10.1186/s12876-016-0562-9. https://doi.org/10.1186/s12876-016-0562-9

15. Samarakoon YM. Risk factors and risk prediction of colorectal cancer among adults in the districts of Colombo and Gampaha.MD Thesis. Faculty of Medicine, University of Colombo, Sri Lanka. 2013.

16. Li J, Chang J, Zhu Y, Yang Y, Gong Y, Ke J, et al. Risk prediction of colorectal cancer with common genetic variants and conventional non-genetic factors in a Chinese Han population. Europe PMC. 2015; 36(10):1053-1057.

17. Jung KJ, Won D, Jeon C, Kim S, Kim T, Jee S. et al. A colorectal cancer prediction model using traditional and genetic risk scores in Koreans. BMC Genet. 2015; 16:49. DOI 10.1186/s12863-015-0207-y https://doi.org/10.1186/s12863-015-0207-y

18. Schroy III PC, Wrong JB, O'Brien MJ, Chen CA, Griffith JL. A Risk Prediction Index for Advanced Colorectal Neoplasia at Screening Colonoscopy. Am J Gastroenterol. 2015;110(7): 1062-1071. doi:10.1038/ajg.2015.146. https://doi.org/10.1038/ajg.2015.146

19. Steffen A, Maclnnis RJ, Joshy G, Giles GG, Banks E, Roder D. Development and Validation of a Risk Score Predicting Risk of Colorectal Cancer. Cancer Epidemiol 
Journal of the Postgraduate Institute of Medicine 2017; 4 (2): E56 1- 24

http://doi.org/10.4038/jpgim.8168

Biomarkers Prev. 2014; DOI: 10.1158/1055-9965.EPI-14-0206.

https://doi.org/10.1158/1055-9965.EPI-14-0206

20. Shin A, Joo J, Yang HR, Bak J, Park Y, Kim J, et al. Risk prediction model for colorectal cancer: national health insurance corporation study, Korea. PLoS One 2014;9:e88079. https://doi.org/10.1371/journal.pone.0088079 PMid:24533067 PMCid:PMC3922771

21. Tao S, Hoffmeister M, BrennerH. Development and validation of a scoring system to identify individuals at high risk for advanced colorectal neoplasms who should undergo colonoscopy screening. Clin Gastroenterol Hepatol. 2014; 12:478-85. https://doi.org/10.1016/j.cgh.2013.08.042 PMid:24022090

22. Wells BJ, Kattan MW, Cooper GS, Jackson L, Koroukian S. Colorectal cancer predicted risk online (CRC-PRO) calculator using data from the multi-ethnic cohort study. J Am Board Fam Med. 2014; 27:42-55. https://doi.org/10.3122/jabfm.2014.01.130040 PMid:24390885 PMCid:PMC4219857

23. Chen G, Mao B, Pan Q, Liu Q, Xu X, Ning Y. Prediction rule for estimating advanced colorectal neoplasm risk in average-risk populations in southern Jiangsu Province. Chin J Cancer Res. 2014; 26:4-11.

PMid:24653621 PMCid:PMC3937762

24. Kaminski MF, Polkowski M, Kraszewska E, Rupinski M, Butruk E, Regula J. A score to estimate the likelihood of detecting advanced colorectal neoplasia at colonoscopy. Gut. 2014; 63:1112-9.

https://doi.org/10.1136/gutjnl-2013-304965 PMid:24385598 PMCid:PMC4078748

25. Stegeman I, de Wijkerslooth TR, Stoop EM, van Leerdam ME, Dekker E, van Ballegooijen $\mathrm{M}$, et al. Combining risk factors with faecal immunochemical test outcome for selecting CRC screening for colonoscopy. Gut. 2014;63: 466-71. https://doi.org/10.1136/gutjnl-2013-305013 PMid:23964098

26. Stegeman I, de Wijkerslooth TR, Stoop EM, van Leerdam ME, Dekker E, van Ballegooijen $\mathrm{M}$, et al. Colorectal cancer risk factors in the detection of advanced adenoma and colorectal cancer. Cancer Epidemiol. 2013;37: 278-83. https://doi.org/10.1016/j.canep.2013.02.004 PMid:23491770

27. Dunlop MG, Tenesa A, Farrington SM, Ballereau S, Brewster DH, Koessler T, et al. Cumulative impact of common genetic variants and other risk factors on colorectal cancer risk in 42103 individuals. Gut. 2012; 62:871-81. https://doi.org/10.1136/gutjnl-2011-300537 PMid:22490517 PMCid:PMC5105590

28. Wrong MCS, Lam TYT, Soi KKFT, Hirai HW, Chan VCW, Ching JYL, et al. A validated tool to predict colorectal neoplasia and inform screening choice for asymptomatic subjects. BMJ Gut. http://dx.doi.org/10.1136/gutjnl-2013-305639. https://doi.org/10.1136/gutjnl-2013-305639

29. Hassan C, Pooler BD, Kim DH, Rinaldi A, Repici A, Pickhardt PJ. Computed tomographic colonography for colorectal cancer screening: risk factors for the 
detection of advanced neoplasia. Cancer. 2013;119:2549-54.

https://doi.org/10.1002/cncr.28007 PMid:23754679 PMCid:PMC3700619

30. Johnson CM, Wei C, Ensor JE, Smolenski DJ, Amos Cl, Levin B, et al. Metaanalyses of colorectal cancer risk factors. Cancer Causes Control. 2013; 24:1207-22. https://doi.org/10.1007/s10552-013-0201-5 PMid:23563998 PMCid:PMC4161278

31. Yarnall JM, Crouch DJM, Lewis CM. Incorporating non-genetic risk factors and behavioural modifications into risk prediction models for colorectal cancer. Cancer Epidemiol. 2013; 37:324-9. https://doi.org/10.1016/j.canep.2012.12.008 PMid:23375517

32. Wang HM, Chang TH, Lin FM, Chao TH, Huang WC, Liang C, et al. A new method for post Genome-Wide Association Study (GWAS) analysis of colorectal cancer in Taiwan. Gene. 2013; 518:107-13. https://doi.org/10.1016/j.gene.2012.11.067 PMid:23262349

33. Lin Y, Yu M, Wang S, Chappell R, Imperiale TF. Advanced colorectal neoplasia risk stratification by penalized logistic regression. Stat Methods Med Res. 2013; 0:0-15.

34. Lubbe SJ, Di Bernardo MC, Broderick P, Chandler I, Houlston RS. Comprehensive evaluation of the impact of 14 genetic variants on colorectal cancer phenotype and risk. Am J Epidemiol. 2012; 175:1-10. https://doi.org/10.1093/aje/kwr285 PMid:22156018

35. Jo J, Nam CM, Sull JW, Yun JE, Kim SY, Lee SJ, et al. Prediction of colorectal cancer risk using a genetic risk score: The Korean Cancer Prevention Study-II (KCPS-II). Genomics Inform. 2012; 10:175. https://doi.org/10.5808/GI.2012.10.3.175 PMid:23166528 PMCid:PMC3492653

36. Cai QC, Da Yu E, Xiao Y, Bai WY, Chen X, He LP, et al. Derivation and validation of a prediction rule for estimating advanced colorectal neoplasm risk in average-risk Chinese. Am J Epidemiol. 2012;175: 584-93. https://doi.org/10.1093/aje/kwr337 PMid:22328705

37. Yeoh K-G, Ho K-Y, Chiu H-M, Zhu F, Ching JYL, Wu D-C, et al. The Asia-Pacific Colorectal Screening score: a validated tool that stratifies risk for colorectal advanced neoplasia in asymptomatic Asian subjects. Gut. 2011; 60:1236-41. https://doi.org/10.1136/gut.2010.221168 PMid:21402615

38. Taylor DP, Stoddard GJ, Burt RW, Williams MS, Mitchell JA, Haug PJ, et al. How well does family history predict who will get colorectal cancer? Implications for cancer screening and counseling. Genet Med. 2011;13: 385-91. https://doi.org/10.1097/GIM.0b013e3182064384 PMid:21270638

39. Marshall KW, Mohr S, El Khettabi F, Nossova N, Chao S, Bao W, et al. A bloodbased biomarker panel for stratifying current risk for colorectal cancer. Int J Cancer. 2010; 126:1177-86. PMid:19795455

40. Ma E, Sasazuki S, Iwasaki M, Sawada N, Inoue M. 10-Year risk of colorectal cancer: development and validation of a prediction model in middle-aged Japanese men. 
Journal of the Postgraduate Institute of Medicine 2017; 4 (2): E56 1- 24

http://doi.org/10.4038/jpgim.8168

Cancer Epidemiol. 2010; 34:534-41.

https://doi.org/10.1016/j.canep.2010.04.021 PMid:20554262

41. Bener A, Moore MA, Ali R, El Ayoubi HR. Impacts of family history and lifestyle habits on colorectal cancer risk: a case-control study inQatar. Asian Pacific J Cancer Prev. 2010; 11:963-8. PMid:21133608

42. Wei EK, Colditz GA, Giovannucci EL, Fuchs CS, Rosner BA. Cumulative risk of colon cancer up to age 70 years by risk factor status using data from the nurses' health study. Am J Epidemiol. 2009; 170:863-72. https://doi.org/10.1093/aje/kwp210 PMid:19723749 PMCid:PMC2800259

43. Almurshed KS. Colorectal cancer: case-control study of sociodemographic, lifestyle and anthropometric parameters in Riyadh. East Mediterr Heal J. 2009; 15:817-26. PMid:20187533

44. Kastrinos F, Allen JI, Stockwell DH, Stoffed EM, Cook EF, Mutinga ML, Balmana J, Syngal S. Development and Validation of a Colon Cancer Risk Assessment Tool for Patients Undergoing Colonoscopy. Am J Gastroenterol. 2009; 104:1508-1518; doi:10.1038/ajg.2009.135 https://doi.org/10.1038/ajg.2009.135

45. Freedman AN, Slattery ML, Ballard-Barbash R, Willis G, Cann BJ, Pee D, et al. Colorectal cancer risk prediction tool for white men and women without known susceptibility. J Clin Oncol. 2009; 27:686. https://doi.org/10.1200/JCO.2008.17.4797 PMid:19114701 PMCid:PMC2645090

46. Liu YC, Sung FC, Hsieh LL, Tang R, Yeh CC. The risk prediction model and risk index for colorectal cancer. Taiwan Journal of Public Health. 2008; 27(1): 1-12.

47. Han M, Choong TL, Hong WZ, Chao S, Zheng R, Kok TY, et al. Novel bloodbased, five-gene biomarker set for the detection of colorectal cancer. Clin Cancer Res. 2008; 14:455-60. https://doi.org/10.1158/1078-0432.CCR-07-1801 PMid:18203981

48. Driver JA, Gaziano JM, Gelber RP, Lee IM, Buring JE, Kurth T. Development of a Risk Score for Colorectal Cancer in Men. Am. J. Med. 2007; 120(3), 257-263. https://doi.org/10.1016/j.amjmed.2006.05.055 PMid:17349449

49. Lin OS, Kozarek RA, Schembre DB, Ayub K,Gluck M, Cantone N, et al. Risk stratification for colon neoplasia: screening strategies using colonoscopy and computerized tomographic colonography. Gastroenterology. 2006; 131:1011-9. https://doi.org/10.1053/j.gastro.2006.08.015 PMid:17030171

50. Wei EK, Giovannucci E, Wu K, Rosner B, Charles S, Willett WC, et al. Comparison of risk factors for colon and rectal cancer. Int J Cancer. 2004; 108:433-42. https://doi.org/10.1002/ijc.11540 PMid:14648711 PMCid:PMC2903217

51. Imperiale TF, Wagner DR, Lin CY, Larkin GN, Rogge JD, Ransohoff DF. Using Risk for Advanced Proximal Colonic Neoplasia To Tailor Endoscopic Screening for Colorectal Cancer. Ann Intern Med. 2003;139(12):959-965. https://doi.org/10.7326/0003-4819-139-12-200312160-00005 PMid:14678915 
52. Betes M, Mu noz-Navas MA, Duque JM, Ang os R, Macías E, S ubtil JC, et al. Use of colonoscopy as a primary screening test for colorectal cancer in average risk people. Am J Gastroenterol. 2003; 98:2648-54. https://doi.org/10.1111/j.1572-0241.2003.08771.x https://doi.org/10.1016/j.amjgastroenterol.2003.09.041 PMid:14687811

53. Camp NJ, Slattery ML. Classification tree analysis: a statistical tool to investigate risk factor interactions with an example for colon cancer (United States). Cancer Causes Control. 2002; 13:813-23. https://doi.org/10.1023/A:1020611416907

54. Colditz GA, Atwood KA, Emmons K, Monson RR, Willett WC, TrichopoulosD, et al. Harvard report on cancer prevention volume 4: Harvard Cancer Risk Index. Risk Index Working Group, Harvard Center for Cancer Prevention. Cancer Causes Control. 2000; 11:477-88. https://doi.org/10.1023/A:1008984432272 https://doi.org/10.1023/A:1008942707187 https://doi.org/10.1023/A:1008982121761 PMid:10880030 